\title{
A narrative review of electroencephalogram-based monitoring during cardiovascular surgery
}

\author{
Heiko A. Kaiser ${ }^{\mathrm{a}}$, Darren Hight ${ }^{\mathrm{a}}$, and Michael S. Avidan ${ }^{\mathrm{b}}$
}

\begin{abstract}
Purpose of review
The current narrative review focuses on depth of hypnosis monitoring with electroencephalography (EEG) during cardiovascular surgery. There have been important findings in recent years regarding the challenges and limitations of EEG-based monitoring during general anesthesia. The purpose of this review is to summarize key EEG-related concepts, as well as to highlight some of the advantages and disadvantages of processed and unprocessed EEG monitoring, especially for older patients with comorbidities undergoing cardiovascular surgery.

\section{Recent findings}

The brain is the target organ of anesthesia. Using the EEG or processed EEG to guide anesthetic administration during cardiovascular surgery conceptually allows precision patient-centered anesthesia. It is suggested that inadequate anesthesia, with the possibility of traumatic intraoperative awareness, can potentially be avoided. Furthermore, excessive anesthesia, with hemodynamic compromise and theoretical risk of delirium, can be minimized. Frail, older patients undergoing major surgery with preexisting neurocognitive disorders might be especially vulnerable to perioperative neurological and other complications. Tailoring anesthetic administration, based on individual patient needs partly guided by certain EEG features, might yield improved perioperative outcomes.
\end{abstract}

\section{Summary \\ Ability to interpret the EEG during surgery might help anesthesia clinicians to individualize anesthetic administration to prevent adverse events, and optimize postoperative recovery.}

Keywords

awareness, cardiovascular anesthesia, delirium, electroencephalogram, intraoperative monitoring

\section{INTRODUCTION}

The role of electroencephalography (EEG)-based brain monitoring in guiding optimal administration of hypnotic agents during general anesthesia has been investigated for many years. Although the EEG shows predictable shifts in activity in response to increasing anesthetic concentrations, the evidence for improved clinical outcomes is conflicting [1-4]. In some countries, EEG monitoring during surgery is common, whereas in others it is rare. For example, in the United Kingdom, the minority of anesthesiologists use intraoperative EEG monitoring, even for high-risk cases [5]. Several organizations have recommended that EEG should be used routinely, especially for patients who are at risk of complications like awareness and delirium [6-8].

The cardiovascular surgery patient population, often older and with multiple comorbidities, is known to be vulnerable to a wide variety of negative clinical outcomes, including intraoperative awareness with recall [9], major organ injury [10], neurocognitive disorders (postoperative delirium, delayed neurocognitive recovery, and minor and major postoperative neurocognitive disorders) [11] and postoperative mortality $[12,13]$. In association with this high vulnerability, specific changes have been reported in the EEG both during wakefulness and with general anesthesia $\left[14^{*}, 15,16\right]$. Furthermore, for these patients, an increased sensitivity to commonly used anesthetics has been described. One of the manifestations of this heightened brain sensitivity is the appearance of EEG burst suppression - epochs of isoelectric EEG punctuated by bursts of activity - at relatively low

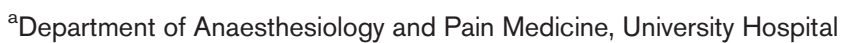
Bern, Inselspital, Bern, Switzerland and ' ${ }^{\mathrm{D}}$ Department of Anesthesiology, Washington University in St. Louis, St. Louis, Missouri, USA

Correspondence to Michael S. Avidan, Department of Anesthesiology, Washington University in St. Louis, St. Louis, Missouri, USA.

Tel: +1 314747 4155; e-mail: avidanm@wustl.edu
}

Curr Opin Anesthesiol 2020, 33:92-100

DOI:10.1097/ACO.0000000000000819 


\section{KEY POINTS}

- The cardiovascular surgery patient population, often older and with multiple comorbidities, is known to be vulnerable to a wide variety of negative clinical outcomes, including intraoperative awareness with recall, major organ injury, neurocognitive disorders, and postoperative mortality.

- Changes in patients' brain electrical activity in response to the administration of general anesthetics might provide information beyond hypnotic depth; indeed, these changes might prove helpful in predicting postoperative outcomes.

- Side effects of anesthetics necessitate their judicious use in patients with cardiovascular disease, especially when they are frail. Monitoring the brain, which is the target organ of general anesthesia, theoretically allows precision anesthesia minimizing side effects such as burst suppression and hypotension.

- The limitations of processed EEG (pEEG) indices are accentuated in patients undergoing cardiovascular surgery, where hypovolemia, low cardiac output, hypotension, hypothermia, acid-base and electrolyte abnormalities, hypoglycemia, cerebral ischemia, and seizures can all lead to changes in the EEG and $p E E G$ indices that might be independent of anesthetic agents or hypnotic depth.

- Lower frontal alpha power during anesthesia, as occurs with older age and with frailty/comorbidities, relative to higher frequency bands might be falsely translated into a higher $\mathrm{pEEG}$ index reading despite lower MAC requirements. This can lead to excessive anesthetic administration to vulnerable older patients.

concentrations of volatile agents or Propofol $[17,18]$. Intraoperative burst suppression with concomitant hypotension has been identified as being associated with increased postoperative mortality [19]. Older age, frailty, and comorbidities might explain this observed increased sensitivity $\left[16,20^{-}\right]$. Based on these observations, it is possible that changes in patients' brain electrical activity in response to the administration of general anesthetics might provide information beyond hypnotic depth; indeed, these changes might prove helpful in predicting postoperative outcomes.

Some EEG changes are not reflected in current processed EEG (pEEG) monitors [e.g., the bispectral index (Medtronic, Minneapolis, Minnesota, USA), SedLine (Masimo, Irvine, California, USA), Entropy (GE Healthcare, Chicago, Illinois, USA), or Narcotrend monitors (MonitorTechnik, Bad Bramstedt, Germany)], which use proprietary algorithms to analyze the EEG signal and generate a number, typically between zero and 100 , intended to reflect the depth of hypnosis. There are many limitations to pEEG indices, to which we will refer in this review. Traditional nonproprietary ways of viewing the EEG include examination of the raw EEG waveform, tracking changes in amplitude over time. Moreover, spectral views of the EEG transformed into the frequency domain can be useful, where power (amplitude squared) is shown for each frequency band (the spectrum), or power is shown at each frequency band over time (the spectrogram). Being able to interpret the changes in EEG and its spectrogram during general anesthesia for cardiac surgery and other procedures can provide information regarding hypnotic depth; acute hypoperfusion of the brain or of one hemisphere (Fig. 1); epileptic discharges; severe brain damage; adequate cooling of the brain before hypothermic circulatory arrest; and return of function after following hypothermic circulatory arrest (Fig. 2).

\section{PRECISION ANESTHESIA}

Older patients comprise a growing number of patients presenting for cardiovascular surgery, and a substantial portion of these patients have numerous comorbidities that might lead to frailty, which translates into decreased cardiopulmonary reserve. Frailty is a central concern for surgical patients, especially in cardiac surgery. The opportunities for both surgical and nonsurgical management of cardiac disease have increased, from minimally invasive interventions like transcatheter aortic valve replacement or mitral clips, to cardiac surgery with small left ventricular assist devices. So, there are viable treatment options even for the frailest of our patients, and we are confronted with difficult decisions regarding anesthetic management. We must balance the side effects of anesthetic agents, with the possible benefits they provide in the face of tissue trauma, painful stimuli, and stress.

The negative inotropic and vasodilatory effects of anesthetics have been well described, as have the cardioprotective properties of volatile anesthetic agents $[21,22]$. Despite the hypothesized protective properties, side effects necessitate the judicious use of anesthetic agents in patients with cardiovascular disease, especially when they are frail. Monitoring the brain, which is the target organ of general anesthesia, theoretically allows precision anesthesia. Otherwise stated, by monitoring the brain with the EEG, anesthesia clinicians can tailor the anesthetic administration to the particular needs of the individual patient, thereby avoiding both underdosing and overdosing of general anesthesia, with their attendant complications. 


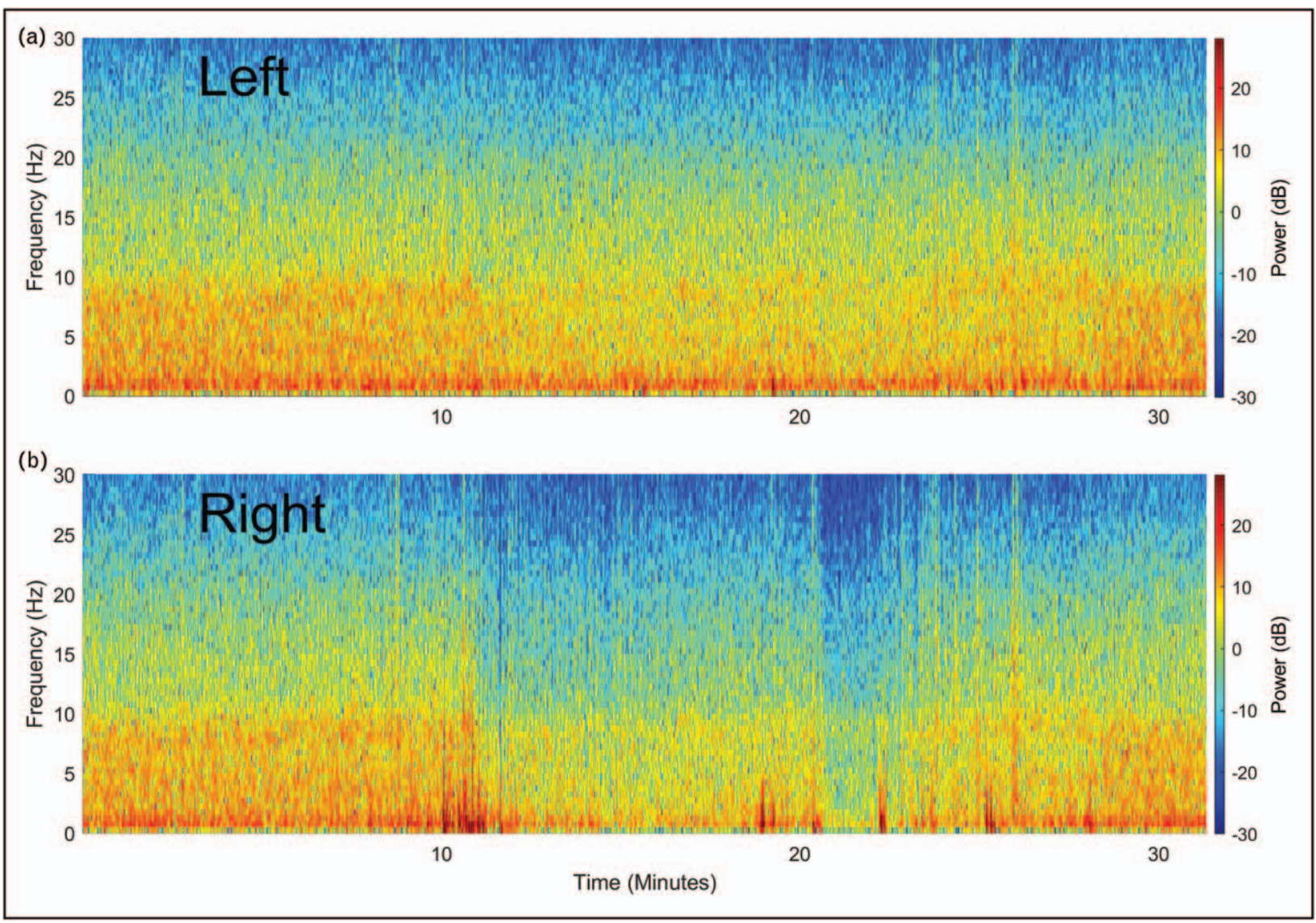

FIGURE 1. Spectrogram in the presence of cerebral hypoperfusion during carotid endarterectomy. The spectrograms show an example electroencephalography response to cortical ischemia during general anesthesia under isoflurane for right-sided carotid endarterectomy. Spectrogram (a) shows electroencephalography recorded from the left hemisphere, with minimal changes, whereas spectrogram (b) from the right hemisphere shows a loss of power (shift away from red) over all frequencies during clamping of the right carotid artery following 10 and $20 \mathrm{~min}$, which recovers before $30 \mathrm{~min}$.

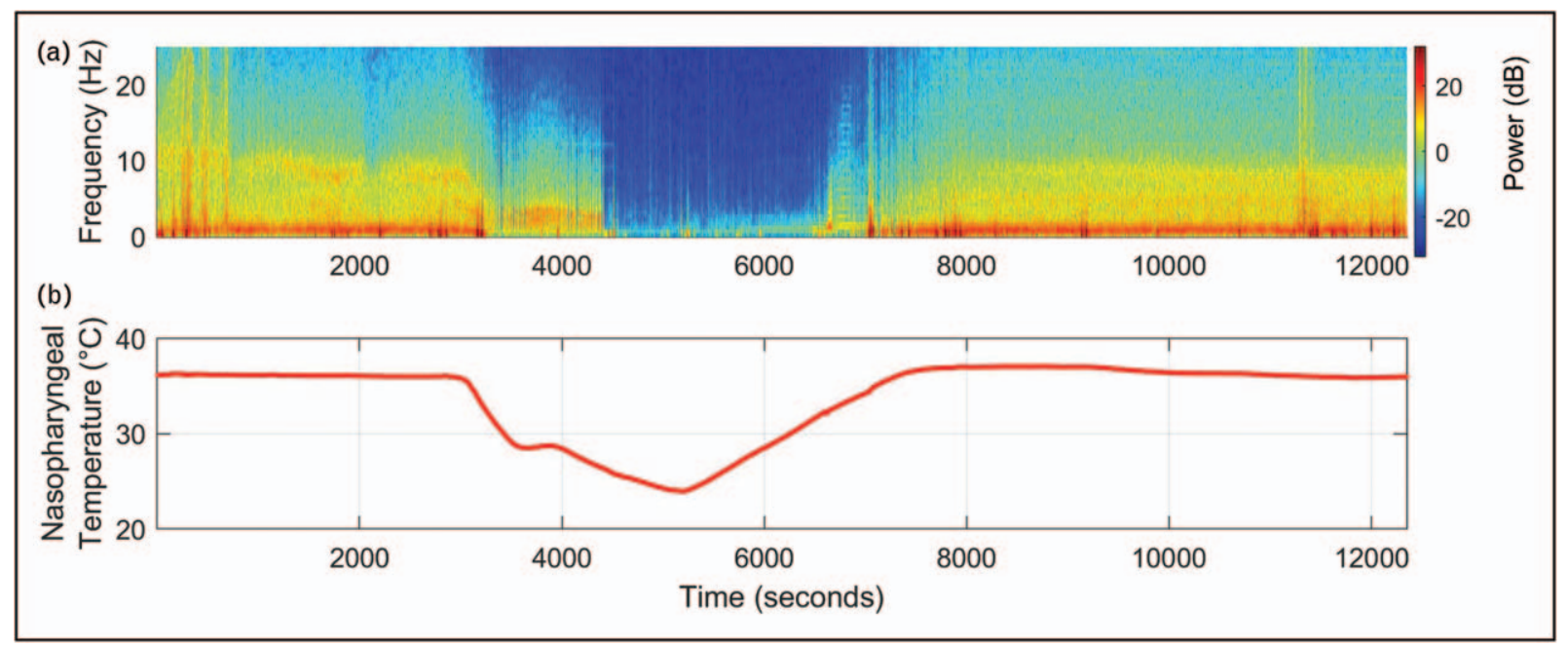

FIGURE 2. Spectrogram during thoracic aortic surgery with deep hypothermic circulatory arrest. The spectrogram (a) is taken from a patient who had aortic arch surgery performed with deep hypothermic circulatory arrest and isoflurane/sufentanil general anesthesia. During cooling (temperature shown in b) burst suppression sets in at $3500 \mathrm{~s}$ with isoelectric electroencephalography starting at $4500 \mathrm{~s}$. During rewarming brain activity recovers with decreased alpha band power initially, which increases steadily until the end of the case. 


\section{ELECTROENCEPHALOGRAPHY CHANGES WITH GENERAL ANESTHESIA}

With increasing volatile or Propofol anesthetic concentrations, the frontally derived EEG changes in well known ways (Fig. 3) [23,24]. The awake EEG shows low-voltage (around $15 \mu \mathrm{V}$, but amplitudes depend on active and reference electrode location) fast activity without distinct activity in a particular frequency band. At anesthetic concentrations resulting in light sedation, the EEG is dominated by oscillations in the beta (around $12-30 \mathrm{~Hz}$ ) frequency band. When concentrations are sufficient to allow surgery, the EEG voltages increase to show larger and slower waveforms (up to $100 \mu \mathrm{V}$ and around $1 \mathrm{~Hz}$, called the slow-wave oscillation) usually overlaid with a second oscillation in the alpha range (classically eight to $12 \mathrm{~Hz}$ ). With further increases in anesthetic concentration, the EEG transitions to burst-suppression, where the EEG shows alternate periods of high amplitude activity (bursts) and low amplitude activity (suppressions, usually less than $5 \mu \mathrm{V}$ ). The length of the suppressed EEG increases with anesthetic concentration, and the EEG can even become entirely suppressed (isoelectric) if anesthetic concentrations are high enough.

(a)
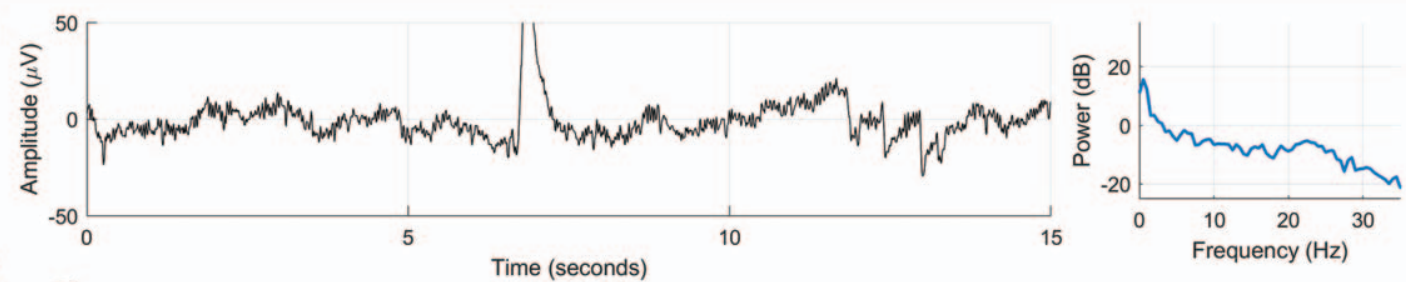

(b)
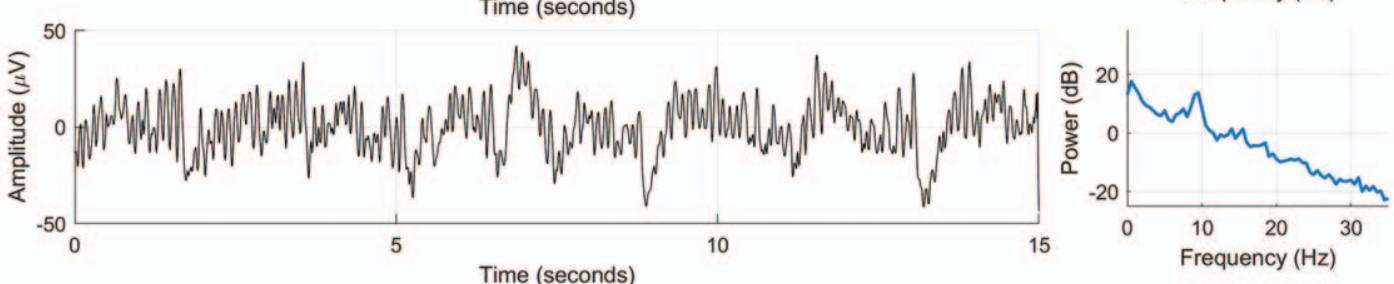

(c)
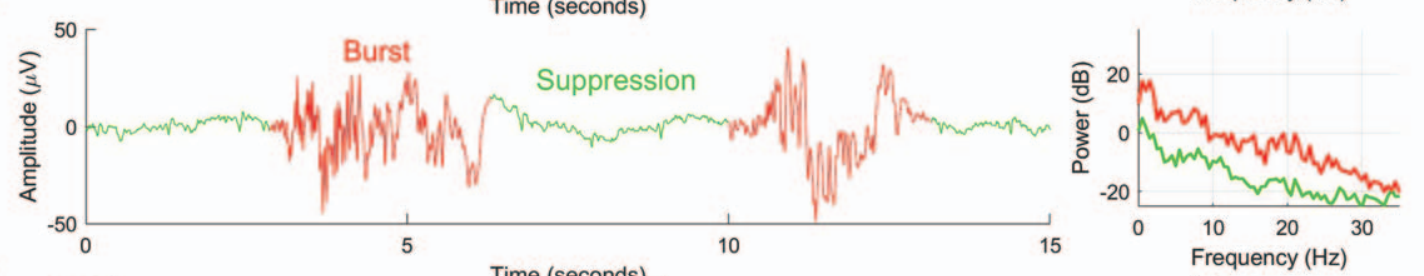

(d)

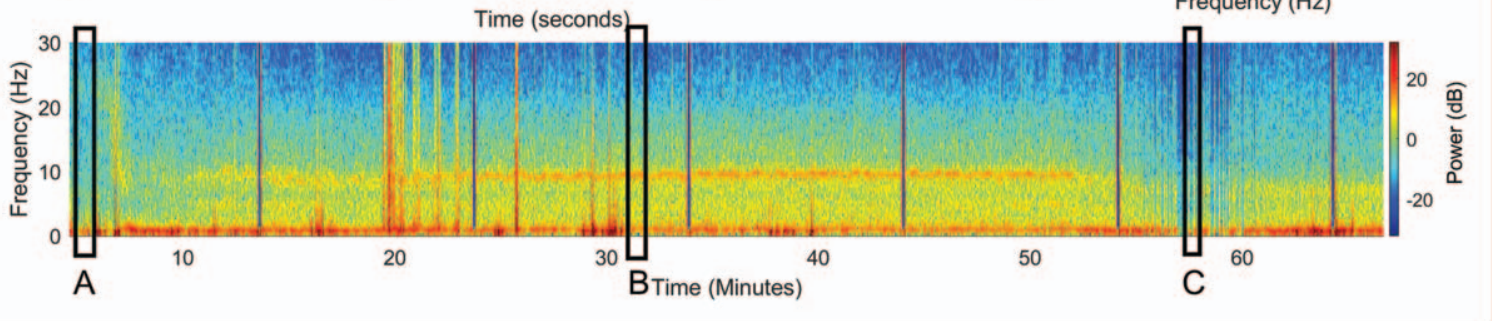

FIGURE 3. Frontal electroencephalography changes during induction and maintenance of anesthesia. This figure shows an example of frontal electroencephalography changes during induction and maintenance of anesthesia with Propofol. In each row raw electroencephalography (voltage over time) is displayed on the left, and the resulting power spectrum (power over frequency) on the right. Row (a) shows an awake electroencephalography prior to induction into anesthesia, with low voltages, and an eye-blink artifact seen at around $7 \mathrm{~s}$. Row (b) shows an electroencephalography during general anesthesia, with a mix of slow-wave (around $1 \mathrm{~Hz}$ ) and alpha (around $9 \mathrm{~Hz}$ ) oscillations. Row (c) shows burst suppression, with burst periods in red and suppression periods in green. Row (d) is a spectrogram where power is displayed as color (with higher power corresponding to red, and lower power corresponding to blue), frequency on the $Y$-axis, and time on the $X$-axis. The location of the example electroencephalography sections in $(a-c)$ are shown with black rectangles. Two kinds of noise are also easily recognizable in the spectrogram: low power sections (blue vertical stripes) where the electroencephalography monitor was completing impedance checks, for example at around $44 \mathrm{~min}$, and high-power sections (red vertical stripes) resulting from electrocautery artifact during surgery (for example at around $20 \mathrm{~min}$ ). 


\section{PREVENTION OF INTRAOPERATIVE AWARENESS}

Cardiac surgical patients have a higher risk than other surgical patients for unintended intraoperative awareness with explicit recall [9]. There are many reasons for this, including the imperative to limit anesthetic administration to minimize hemodynamic side effects. The transition periods when initiating and terminating cardiopulmonary bypass are also likely to represent high-risk times. During commencement of bypass, concentrations of anesthetic and analgesic agents can decrease rapidly through hemodilution as well as absorption by the circuit and the membrane oxygenator. Transitions can also be associated with rapid changes in flow/cardiac output (CO), cerebral blood flow, and temperature, all of which can alter depth of hypnosis during general anesthesia [25]. Furthermore, during these transitions, clinicians (either the anesthesiology team or the perfusionists) might forget to initiate anesthetic agent administration. And when attempting to wean a patient from cardiopulmonary bypass, hemodynamic compromise can curtail the ability to safely institute or increase anesthetic agents.

In patients who are at high risk for intraoperative awareness with recall, EEG-based monitoring with pEEG indices probably decreases the incidence of awareness. However, pEEG monitors are likely no more effective in this regard than instituting a protocol with an alert for low volatile anesthetic concentration [4]. Yet, during cardiopulmonary bypass, monitoring volatile anesthetic agent concentration can be challenging. Therefore, clinicians often use total intravenous anesthesia (TIVA) alone or to complement the volatile anesthetic. For TIVA, there is evidence to suggest that pEEG indices are helpful in preventing awareness with recall $[3,26]$. There is a strong argument to be made that whenever TIVA is administered in combination with neuromuscular blocking agents, monitoring the brain with EEG or a pEEG monitor is mandatory. The fundamental challenge in relation to such brain monitoring is the problem of calibration. Since there is no gold standard measure for depth of hypnosis beyond loss of responsiveness, the accuracy of EEG and pEEG devices in tracking depth of hypnosis cannot currently be determined. This challenge was emphasized recently in a study demonstrating that some patients who, during intended general anesthesia, had a specific EEG pattern (frontal delta waves phased amplitude coupled with alpha spindles) thought to indicate unconsciousness; yet $7 \%$ responded appropriately to commands with the isolated forearm technique suggesting that they were in fact conscious [27"].

\section{CHALLENGES IN MONITORING HYPNOTIC} DEPTH

Currently available pEEG monitors [e.g. the bispectral index (Medtronic), SedLine (Masimo), Entropy (GE Healthcare) or Narcotrend monitors (MonitorTechnik, Bad Bramstedt, Germany) use proprietary algorithms to transform data from the EEG into a linear scale from 0 to 100 , were zero represents absence of detectable brain electrical activity (persistent suppression), and values close to 100 are consistent with arousal states. Values between about 40 and 60 indicate a hypnotic state (e.g. sleep or general anesthesia), with lower values suggesting deep anesthesia, and higher values suggesting sedation. There are several problems with this representation, including the following assumptions: hypnotic depth with general anesthesia follows a linear path; the paths of increasing and decreasing hypnotic depths are similar, but just in opposite directions; that these numbers have neurobiological meaning and are comparable among different patients regardless of age, sex, or combination of drugs administered. All three of these assumptions are likely to be flawed. First, various investigators have shown that there are likely to be stable neurological states, and transitions between them are often abrupt and might not be bidirectional [28"-]. A recent human volunteer study also strongly suggests that depth of hypnosis or anesthesia is not a simple linear process. In this study, volunteers were deeply anesthetized with isoflurane for over $3 \mathrm{~h}$. Regardless of how much time the EEG showed suppression or where the suppression occurred temporally, there was no association between any suppression parameter and the time to emerge from anesthesia [29"']. So even with EEG criteria indicative of very deep anesthesia, emergence on cessation of anesthetic administration could be rapid or slow; the transition time from suppressed to aroused EEG was not uniform across volunteers. The absence of smooth transitions is an important barrier to using pEEG indices to guide fine or precise titration of anesthetic agents [30]. Second, the phenomenon of neural inertia has been demonstrated in several species, and is likely to apply in humans as well. This phenomenon refers to the observation that higher concentrations of hypnotic agents are required in individual animals to achieve loss of responsiveness compared with the concentration at which return of responsiveness occurs. This is likely not just a pharmacokinetic confound, but rather suggests that different neurobiological mechanisms or pathways might be involved in the induction and emergence processes [31,32]. Third, there have been several studies that show that pEEG indices are affected by multiple factors including 
surgical stimulation, regional anesthesia, age, comorbidity, preexisting cognitive impairment, sex, temperature, anesthetic agent, and combination of drugs [33]. It should be obvious to most clinicians that a bispectral index value of 50 in a healthy 30-year-old woman receiving intravenous propofol with remifentanil for an appendectomy cannot have the same neuro-biological meaning as a bispectral index value of 50 in a frail 80-yearold man who is receiving an opioid-heavy volatile anesthetic for an aortic valve replacement.

The limitations of pEEG indices are accentuated in patients undergoing cardiovascular surgery, where hypovolemia, low CO, hypotension, hypothermia, acid-base and electrolyte abnormalities, hypoglycemia, cerebral ischemia, and seizures can all lead to changes in the EEG and pEEG indices that might be independent of anesthetic agents or hypnotic depth [34"]. Age and comorbidities are associated with a decrease in minimum alveolar concentration (MAC) as well as frontal alpha power. Frontal alpha oscillations during general anesthesia are a useful sign of unconsciousness; with loss of consciousness, anteriorization of alpha oscillations occurs (i.e., a shift from occipital to frontal oscillations) [35]. But a lower frontal alpha power during anesthesia, as occurs with older age and with frailty/comorbidities, relative to higher frequency bands might be falsely translated into a higher pEEG index reading despite lower MAC requirements. This can lead to excessive anesthetic administration to vulnerable older patients [36]. This hypothesis is supported by a large retrospective analysis reporting paradoxically higher bispectral index values, despite higher (than necessary) age-adjusted MAC fractions [37"']. As displayed in Fig. 4, different pEEG algorithms interpret a given frontal EEG quite differently, so this issue might be more relevant with the bispectral index algorithm. Effects of comorbidities on pEEG indices or algorithms have not been well investigated to date.

\section{PATIENT CHARACTERISTICS}

Increasing patient age is associate with various alterations in the EEG during general anesthesia. The frequency and power of the alpha oscillation

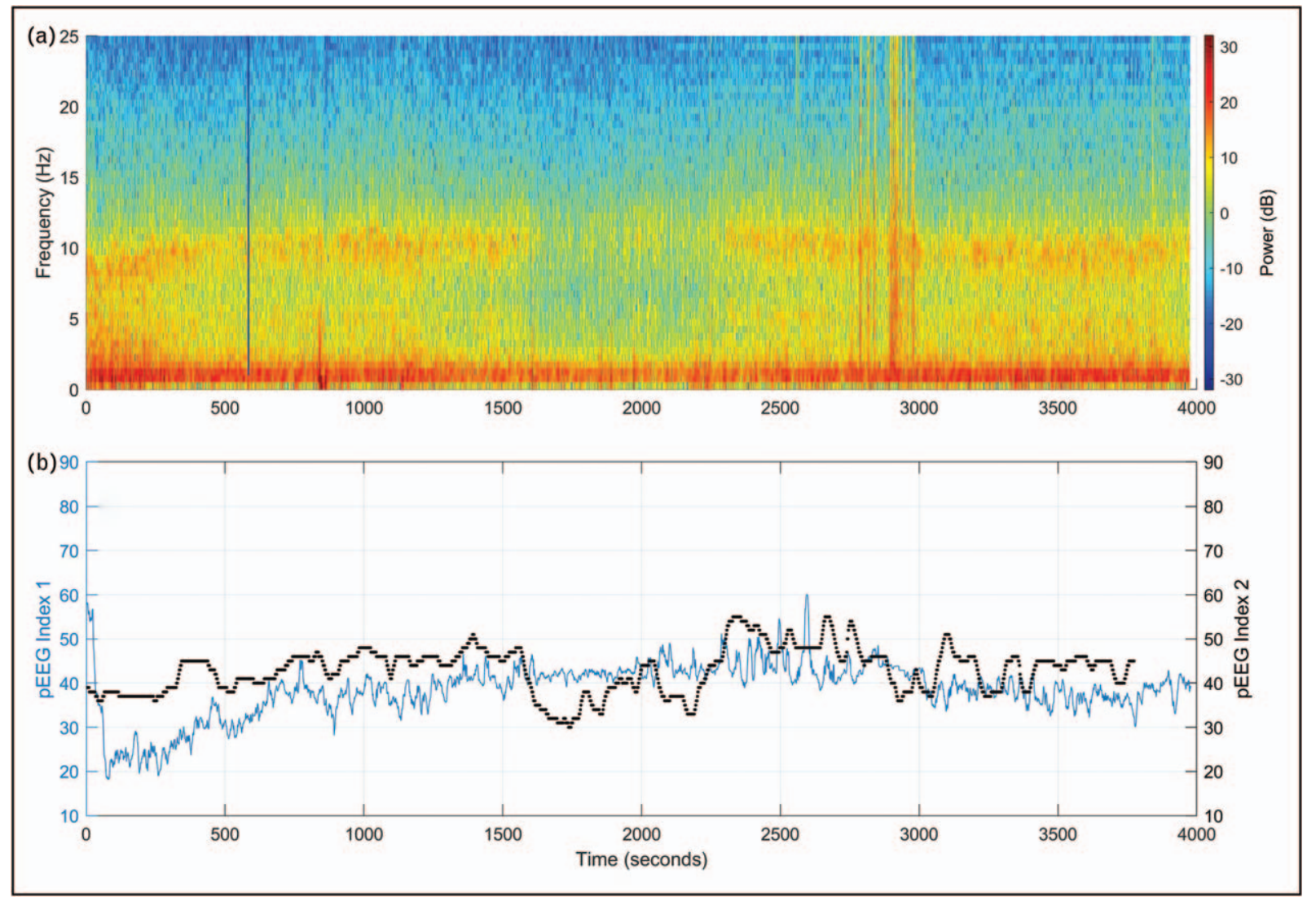

FIGURE 4. Spectrogram depicting lightening of anesthesia and two derived electroencephalography indices. The spectrogram (a) shows an example of unintended inadequate isoflurane anesthesia during separation from cardiopulmonary bypass. Increasing peak alpha frequency (0-1500 s) with loss of alpha power at $1600 \mathrm{~s}$ are spectral signatures of arousal [38"]. The concurrent processed electroencephalography indices (b) illustrate that some current processed electroencephalography algorithms do not depict this arousal event; neither index increases markedly after $1500 \mathrm{~s}$. 
(approximately between 8 and $12 \mathrm{~Hz}$ ) decreases with both increasing age and with increasing volatile anesthetic concentration [20",39]. Age is also known to be associated with decreased overall EEG power in all frequency bands, apart from just alpha [39]. Providing precision anesthesia to older patients is challenging for several reasons. First, older adults require lower concentrations of both volatile agents and Propofol to achieve adequate hypnotic depth, but there is likely to be wider interpatient variability than with young, healthy patients. Second, older patients are often more vulnerable to the cardiorespiratory side effects of anesthetic agents, including hypotension. This can make it difficult to safely administer adequate anesthetic concentrations. Third, as noted, the EEG changes that occur during general anesthesia are different in older adults, but there is also more interpatient variability. Furthermore, the EEG changes in older adults during anesthesia have not been as well characterized as they have been in younger adults. Many of the studies describing EEG changes have been conducted in young, healthy patients or volunteers. The variability, both in relation to anesthetic requirements and EEG changes in older adults, probably relates to factors such as variability in preexisting neurodegenerative disorders, comorbidities and patient frailty.

Although these are difficult to study, various (age-related) diseases are associated with notable EEG changes during general anesthesia. For example, with deteriorating health, there is increasing sensitivity to anesthetic agents. This is reflected in the EEG by less prominent or slow alpha oscillations, as well as the appearance of EEG suppression at low anesthetic concentrations [16]. In general, owing to obvious challenges, there has been little focused work on the influence of specific comorbidities on the EEG during general anesthesia. The importance of clarifying the impact of specific diseased on the EEG was emphasized in research conducted by Wu et al. [40].

\section{BURST SUPPRESSION AND PERSISTENT SUPPRESSION}

Burst suppression and persistent suppression are not physiological EEG features. They can occur during excessively deep general anesthesia and in pathological states like traumatic brain injury, coma, severe hypothermia, hypoxia, hypoglycemia, encephalopathies, or hypoperfusion of the brain [41]. This is especially relevant during cardiovascular surgery, excessively deep general anesthesia could be mimicked by different pathological states, including hypotension, low $\mathrm{CO}$, or stroke. In these situations, both EEG features and processed EEG readings will suggest that anesthesia is excessive, whereas the actual explanation might be more sinister requiring immediate intervention. Regardless of whether EEG suppression or burst suppression are themselves injurious, it is reasonable to address several of the potential contributors to EEG suppression [including interventions such as decreasing anesthetic administration, increasing blood pressure (BP), and active warming when hypothermia is not required], since it is unlikely in most instances (apart from status epilepticus) for EEG suppression to be therapeutic.

Intraoperative hypotension is a modifiable risk factor for perioperative stroke during cardiac surgery [42], and in noncardiac surgery, associations between hypotension on the one hand and delirium, acute kidney injury, myocardial injury and mortality on the other have been reported [43]. The relevance to the EEG is that hypotension is also associated with EEG changes during general anesthesia, such as EEG suppression $[44,45]$. In attempting to ensure adequate anesthesia, overdosing with attendant hypotension can readily occur. Theoretically, using the EEG or processed EEG as a titration aid can allow clinicians to administer just the right amount of anesthetic, thereby avoiding EEG suppression and minimizing side effects such as hypotension. On the contrary, precise titration of anesthesia guided by the EEG might not feasible for several reasons, including depth of anesthesia probably does not follow smooth or predictable course [46]; the anesthetic concentration required to induce anesthesia might be different from the concentration at which responsiveness returns [32]; and the suggested EEG and processed EEG correlates of unconsciousness might not actually be $100 \%$ specific for unconsciousness [27",47].

Although several studies have demonstrated a robust and 'dose-response' relationship between EEG suppression and postoperative delirium, the ENGAGES trial did not demonstrate that actively decreasing the duration of EEG suppression during surgery prevented postoperative delirium. Thus, it remains controversial whether or not there is a causal link between intraoperative EEG suppression and postoperative delirium (and other adverse outcomes, like death) [2]. Although there was no difference in the incidence of delirium between the usual care and EEG guided group in the ENGAGES trial, the mortality incidence was four times higher in the usual care group despite similar mean arterial pressures, but with more phenylephrine administration [2]. Furthermore, there was still EEG suppression in the guided group [2], and the concurrence of hypotension and EEG suppression have been associated with postoperative mortality $[19,48]$. Thus, taking together everything that is currently known, it 
remains compelling to monitor both the EEG and BP during surgery, and to avoid both hypotension and EEG suppression whenever feasible. Such an approach might be beneficial and is unlikely to harm patients.

\section{CONCLUSION}

For the many reasons articulated in this review, we strongly recommend the routine use of EEG monitoring during general anesthesia, and especially cardiovascular surgery. Currently pEEG indices are often error prone, and are probably inadequate for preventing the overdosing of older and more frail patients. We believe that understanding EEG and spectrogram changes in a more sophisticated way, such overdosing can more readily be avoided, and, in general, more precise or tailored administration of anesthesia might be achieved. Avoiding EEG suppression is one approach that might be helpful in preventing unnecessarily deep anesthesia, with the likely additional benefit of limiting side effects of general anesthesia, especially hemodynamic instability. We hope that leaders in the specialty will heed the clarion call such that electroencephalography will be one of the core educational competencies for anesthesiologists and other anesthesia clinicians, and that EEG brain monitoring will become standard intraoperative practice.

\section{Acknowledgements}

None.

\section{Financial support and sponsorship}

None.

\section{Conflicts of interest}

There are no conflicts of interest.

\section{REFERENCES AND RECOMMENDED \\ READING}

Papers of particular interest, published within the annual period of review, have been highlighted as:

- of special interest

- of outstanding interest

1. Radtke FM, Franck M, Lendner J, et al. Monitoring depth of anaesthesia in a randomized trial decreases the rate of postoperative delirium but not postoperative cognitive dysfunction. Br J Anaesth 2013; 110(Suppl 1:i):98-105.

2. Wildes TS, Mickle AM, Ben Abdallah A, et al. Effect of electroencephalography-guided anesthetic administration on postoperative delirium among older adults undergoing major surgery. JAMA 2019; 321:473-511.

3. Myles PS, Leslie K, McNeil J, et al. Bispectral index monitoring to prevent awareness during anaesthesia: the B-Aware randomised controlled trial. Lancet $2004 ; 363: 1757-1763$.

4. Avidan MS, Zhang L, Burnside BA, et al. Anesthesia awareness and the bispectral index. N Engl J Med 2008; 358:1097-1108.

5. Pandit JJ, Cook TM, Jonker WR, O'Sullivan E; 5th National Audit Project (NAP5) of the Royal College of Anaesthetists, the Association of Anaesthetists of Great Britain, Ireland. A national survey of anaesthetists (NAP5 baseline) to estimate an annual incidence of accidental awareness during general anaesthesia in the UK. Br J Anaesth 2013; 110:501-509.
6. Smith $D$, Andrzejowski J, Smith A. Certainty and uncertainty: NICE guidance on 'depth of anaesthesia' monitoring. Anaesthesia 2013; 68:1000-1005.

7. American Geriatrics Society Expert Panel on Postoperative Delirium in Older Adults. Postoperative delirium in older adults: best practice statement from the American Geriatrics Society. J Am Coll Surg 2015; 220:136-148.e1.

8. Aldecoa C, Bettelli G, Bilotta F, et al. European Society of Anaesthesiology evidence-based and consensus-based guideline on postoperative delirium. Eur J Anaesthesiol 2017; 34:192-214.

9. Smith D, Goddard NG. Awareness in cardiothoracic anaesthetic practice where now after NAP5? Anaesthesia 2015; 70:130-134.

10. Biccard BM, Scott DJA, Chan MTV, et al. Myocardial injury after noncardiac surgery (MINS) in vascular surgical patients: a prospective observational cohort study. Ann Surg 2018; 268:357-363.

11. Brown $\mathrm{CH}$, Probert J, Healy $\mathrm{R}$, et al. Cognitive decline after delirium in patients undergoing cardiac surgery. Anesthesiology 2018; 129:406-416.

12. Mazzeffi M, Zivot J, Buchman $T$, Halkos M. In-hospital mortality after cardiac surgery: patient characteristics, timing, and association with postoperative length of intensive care unit and hospital stay. Ann Thorac Surg 2014; 97:1220-1225.

13. Pearse RM, Moreno RP, Bauer $P$, et al. Mortality after surgery in Europe: a 7 day cohort study. Lancet 2012; 380:1059-1065.

14. Jefferson $A L$, Thompson $M$. Cardiovascular disease and cognitive aging. In:

- Yaffe K, editor. Chronic medical disease and cognitive aging. Oxford: Oxford University Press; 2018. pp. 1-39.

Excellent book on comorbidities and cognitive changes. Especially relevant for this review is the chapter on cardiovascular disease and its cognitive consequences.

15. Rossini PM, Rossi S, Babiloni C, Polich J. Clinical neurophysiology of aging brain: from normal aging to neurodegeneration. Prog Neurobiol 2007; 83:375-400.

16. Kaiser HA, Hirschi T, Hartwich V, et al. Decreasing frontal electroencephalogram alpha power and increasing sensitivity to volatile anesthetics over 3 surgeries within 7 months: a case report. A A Pract 2019; 13:274-277.

17. Whitlock EL, Torres BA, Lin N, et al. Postoperative delirium in a substudy of cardiothoracic surgical patients in the BAG-RECALL clinical trial. Anesth Analg 2014; 118:809-817.

18. Fritz BA, Kalarickal PL, Maybrier HR, et al. Intraoperative electroencephalogram suppression predicts postoperative delirium. Anesth Analg 2016; 122:234-242.

19. Willingham $M$, Ben Abdallah $A$, Gradwohl $S$, et al. Association between intraoperative electroencephalographic suppression and postoperative mortality. Br J Anaesth 2014; 113:1001-1008.

20. Hight D, Voss LJ, García PS, Sleigh J. Changes in alpha frequency and power

- of the electroencephalogram during volatile-based general anesthesia. Front Syst Neurosci 2017; 11:36.

The publication presents the changes of alpha frequency oscillations due to aging and alterations of anesthetic concentrations very clearly. The information can help to guide dosing of anesthetics in a vulnerable patient population.

21. Khambatta HJ, Sonntag $H$, Larsen $R$, et al. Global and regional myocardial blood flow and metabolism during equipotent halothane and isoflurane anesthesia in patients with coronary artery disease. Anesth Analg 1988; $67: 936-942$.

22. De Hert SG, Turani F, Mathur S, Stowe DF. Cardioprotection with volatile anesthetics: mechanisms and clinical implications. Anesth Analg 2005; 100: $1584-1593$.

23. Bennett C, Voss LJ, Barnard JPM, Sleigh JW. Practical use of the raw electroencephalogram waveform during general anesthesia: the art and science. Anesth Analg 2009; 109:539-550.

24. Purdon PL, Sampson A, Pavone KJ, Brown EN. Clinical electroencephalography for anesthesiologists: Part I: background and basic signatures. Anesthesiology 2015; 123:937-960.

25. Mets $B$. The pharmacokinetics of anesthetic drugs and adjuvants during cardiopulmonary bypass. Acta Anaesthesiol Scand 2000; 44:261-273.

26. Avidan MS, Mashour GA. Prevention of intraoperative awareness with explicit recall: making sense of the evidence. Anesthesiology 2013; 118:449-456.

27. Gaskell AL, Hight DF, Winders J, et al. Frontal alpha-delta EEG does not

- preclude volitional response during anaesthesia: prospective cohort study of the isolated forearm technique. Br J Anaesth 2017; 119:664-673.

The study shows that the widely propagated frontal alpha/delta electroencephalography (EEG) pattern of unconsciousness during general anesthesia might not always signify unconsciousness.

28. Proekt $A$, Hudson $A E$. A stochastic basis for neural inertia in emergence from - general anaesthesia. Br J Anaesth 2018; 121:86-94.

The article presents a perspective that depth of hypnosis is not a linear phenomenon: emergence from anesthesia occurs at a lower anesthetic concentration than induction, and this seems to be independent of pharmacokinetic factors.

29. Shortal BP, Hickman LB, Mak-McCully RA, et al. Duration of EEG suppression

-1 does not predict recovery time or degree of cognitive impairment after general anaesthesia in human volunteers. Br J Anaesth 2019; 123:206-218.

The trial indicates, that the time of emergence is independent of depth of hypnosis at the time of discontinuation of the anesthetic. EEG suppression in itself is not an important determinant of recovery time.

30. Palanca BJA, Mashour GA, Avidan MS. Processed electroencephalogram in depth of anesthesia monitoring. Curr Opin Anaesthesiol 2009; 22:553-559. 
31. Friedman EB, Sun $Y$, Moore JT, et al. A conserved behavioral state barrier impedes transitions between anesthetic-induced unconsciousness and wakefulness: evidence for neural inertia. PLoS One 2010; 5:e11903.

32. Kuizenga $\mathrm{MH}$, Colin PJ, Reyntjens KMEM, et al. Test of neural inertia in humans during general anaesthesia. Br J Anaesth 2018; 120:525-536.

33. Dahaba AA. Different conditions that could result in the bispectral index indicating an incorrect hypnotic state. Anesth Analg 2005; 101:765-773.

34. Dahaba AA. Brain function monitors: are we just 'Chasing' the numbers?

- Anesth Analg 2019; 128:1042-1044.

This is an interesting 'open mind' commentary on why we should not trust or use EEG-based indices anymore to guide general anesthesia.

35. Purdon PL, Pierce ET, Mukamel EA, et al. Electroencephalogram signatures of loss and recovery of consciousness from propofol. Proc Natl Acad Sci U S A 2013; 110:E1142-E1151.

36. Whitlock EL, Sleigh J. Are we overdosing older patients? Br J Anaesth 2019; 123:257-258.

37. Ni K, Cooter M, Gupta DK, et al. Paradox of age: older patients receive higher

- age-adjusted minimum alveolar concentration fractions of volatile anaesthetics yet display higher bispectral index values. Br J Anaesth 2019; 123:288-297

The work by $\mathrm{Ni}$ et al. sheds a critical light on the use of the bispectral index monitor. It hypothesizes and presents evidence that with its use we might be overdosing older patients.

38. Hesse S, Kreuzer M, Hight D, et al. Association of electroencephalogram

- trajectories during emergence from anaesthesia with delirium in the postanaesthesia care unit: an early sign of postoperative complications. $\mathrm{Br} \mathrm{J}$ Anaesth 2019; 122:622-634.

Although the study was aimed at the analysis of associations between different EEG emergence patterns and delirium, it also presents frontal EEG signatures that could signify (unintended) lightening of general anesthesia during maintenance.
39. Purdon PL, Pavone KJ, Akeju O, et al. The ageing brain: age-dependent changes in the electroencephalogram during propofol and sevoflurane general anaesthesia. Br J Anaesth 2015; 115(Suppl 1):i46-i57.

40. $\mathrm{Wu} Y$, Jin $\mathrm{S}$, Zhang $\mathrm{L}$, et al. Minimum alveolar concentration-awake of sevoflurane is decreased in patients with end-stage renal disease. Anesth Analg 2019; 128:77-82.

41. Brown EN, Lydic R, Schiff ND. General anesthesia, sleep, and coma. N Engl J Med 2010; 363:2638-2650.

42. Sun LY, Chung AM, Farkouh ME, et al. Defining an intraoperative hypotension threshold in association with stroke in cardiac surgery. Anesthesiology 2018; 129:440-447.

43. Wesselink EM, Kappen $\mathrm{TH}$, Torn $\mathrm{HM}$, et al. Intraoperative hypotension and the risk of postoperative adverse outcomes: a systematic review. $\mathrm{Br} J$ Anaesth 2018; 121:706-721.

44. Bozorgi A, Chung $S$, Kaffashi $F$, et al. Significant postictal hypotension: expanding the spectrum of seizure-induced autonomic dysregulation. Epilepsia 2013; 54:e127-e130.

45. Stockard JJ, Bickford RG, Myers RR, et al. Hypotension-induced changes in cerebral function during cardiac surgery. Stroke 1974; 5:730-746.

46. Whitlock EL, Villafranca AJ, Lin N, et al. Relationship between bispectral index values and volatile anesthetic concentrations during the maintenance phase of anesthesia in the B-Unaware trial. Anesthesiology 2011; 115:1209-1218.

47. Schuller PJ, Newell S, Strickland PA, Barry JJ. Response of bispectral index to neuromuscular block in awake volunteers. Br J Anaesth 2015; 115(Suppl 1): i95-i103.

48. Kertai MD, Pal N, Palanca BJA, et al., B-Unaware Study Group. Association of perioperative risk factors and cumulative duration of low bispectral index with intermediate-term mortality after cardiac surgery in the B-Unaware Trial. Anesthesiology 2010; 112:1116-1127. 